\title{
On $(-1,1)$-Matrices of Skew Type with the Maximal Determinant and Tournaments
}

\author{
José Andrés Armario
}

Dedicated to Hadi Kharaghani on the occasion on his 70th birthday

\begin{abstract}
Skew Hadamard matrices of order $n$ give the solution to the question of finding the largest possible $n$ by $n$ determinant with entries \pm 1 of skew type when $n \equiv 0(\bmod 4)$. Characterizations of skew Hadamard matrices in terms of tournaments are well-known. For $n \equiv 2(\bmod 4)$, we give a characterization of $(-1,1)$-matrices of skew type of order $n$ where their determinants reach Ehlich-Wojtas' bound in terms of tournaments.
\end{abstract}

Keywords Tournaments • Maximal determinants • Skew $(-1,1)$-matrices

\section{Introduction}

Let $g(n)$ denote the maximum determinant of all $n \times n$ matrices with elements \pm 1 . Here and throughout this paper, for convenience, when we say determinant we mean the absolute value of the determinant. The question of finding $g(n)$ for any integer $n$ is an old one which remains unanswered in general. We ignore here the trivial cases $n=1,2$. In 1893 Hadamard gave the upper bound $n^{n / 2}$ for $g(n)$. This bound can be attained only if $n$ is a multiple of 4. A matrix that attains it is called a Hadamard matrix, and it is an outstanding conjecture that one exists for any multiple of 4 . At the time of writing, the smallest order for which the existence of a Hadamard matrix is in question is 668. If $n$ is not a multiple of $4, g(n)$ is not known in general, but tighter bounds exist. For $n \equiv 2(\bmod 4)$, Ehlich [3] and independently Wojtas [8] proved that

\footnotetext{
J.A. Armario $(\triangle)$

Department of Applied Mathematics I, University of Sevilla, Sevilla, Spain

e-mail: armario@us.es
} 


$$
g(n) \leq(2 n-2)(n-2)^{\frac{1}{2} n-1} \quad(\text { Ehlich-Wojtas' bound })
$$

This bound can be attained only if $2 n-2$ is the sum of two squares. When an $n \times n$ determinant is found that attains the upper bound, it is immediate that the maximal determinant for that order is just the bound itself. From now on, we will call the matrices attaining Ehlich-Wojtas' bound, $E-W$ matrices. It has been conjectured that E-W matrices of order $n$ exist when $2 n-2=\alpha^{2}+\beta^{2}$ for some positive integers $\alpha$ and $\beta$. The interested reader is addressed to [4] and the website [6] for further information on what is known about maximal determinants.

A $(-1,1)$-matrix $M$ of order $n$ is said to be of skew type if $M+M^{T}=2 I$, where $I$ denotes the identity matrix and $M^{T}$ the transpose of $M$. A Hadamard matrix $H$ of skew type is called a skew Hadamard matrix. Whenever a skew $(-1,1)$-matrix is mentioned in this paper, we mean a $(-1,1)$-matrix of skew type.

It was conjectured that skew Hadamard matrices exist for any order multiple of 4. However, it was proved [1] that skew E-W matrices may only exist when $2 n-3=\alpha^{2}$ for some integer $\alpha$ (i.e., $\beta=1$ ), a condition which is believed to be sufficient. In [1] examples of skew E-W matrices for small orders have been provided.

A tournament $T=(V, E)$ of order $n$ is a directed graph where the vertex set $V$ consists of $n$ elements and the edge set $E \subset V \times V$ such that each pair of vertices $x$ and $y$ is joined by exactly one of the edges $(x, y)$ or $(y, x)$. The adjacency matrix $A$ of a directed graph, $G=(V, E)$, is indexed by the vertex set $V$, and its entries are defined as follows:

$$
[A]_{x, y}= \begin{cases}1 & \text { if }(x, y) \in E, \\ 0 & \text { otherwise }\end{cases}
$$

Thus, a directed graph $T$ is a tournament if and only if its adjacency matrix satisfies

$$
A+A^{T}=J-I
$$

where $J$ is the all-ones matrix of order $n$. Throughout this paper we use $A$ for the adjacency matrix of the tournament $T$. The vector $s=A \mathbf{1}$ is called the score vector of the tournament, where $\mathbf{1}$ is the all-ones column vector. Clearly,

$$
\mathbf{1}^{T} s=\left(\begin{array}{l}
n \\
2
\end{array}\right),
$$

and the $i$-th entry of $s$, denote by $s_{i}$, is the outdegree of vertex $i$-th in the tournament $T$. A tournament $T$ of order $n$ is regular if all entries of the score vector are equal to $\frac{(n-1)}{2}$, which implies that $n$ must be odd. A tournament $T$ of even order $n$ is almost regular if the entries of the column vector $A \mathbf{1}$ are $\frac{n}{2}$ and $\frac{(n-2)}{2}$, each appearing $\frac{n}{2}$ times. The tournament $T$ is doubly regular of degree $t$ provided any two 
vertices of $T$ jointly dominate precisely $t$ vertices. It is easy to see that if $T$ is doubly regular, then $T$ is regular with degree $2 t+1$. Thus, $T$ is doubly regular of degree $t$ if and only if $A$ satisfies $A A^{T}=t J_{n}+(t+1) I_{n}$ where $n=4 t+3$. Consequently, $T$ is doubly regular with parameters $(4 t+3,2 t+1, t)$.

Let $e_{x, y}$ denote the edge of $T$ defined by the vertices $x$ and $y . d^{+}\left(e_{x, y}\right)$ denotes the number of vertices dominated by both $x$ and $y$ and $\mathrm{d}^{-}\left(e_{x, y}\right)$ denotes the number of vertices dominating both vertices $x$ and $y$. That is, $d^{+}\left(e_{x, y}\right)$ [respectively $\left.\mathrm{d}^{-}\left(e_{x, y}\right)\right]$ is the number of +1 's (respectively, 0 's) that the rows of $A$ indexed by $x$ and $y$ contain in the same column.

For a $(-1,1)$-matrix of skew type $M$ of order $n$, we normalize $M$ so that the first row of $M$ consists of the all-ones vector with keeping to be of skew type. We can construct a $(0,1)$-matrix $A$ of order $n-1$ as the submatrix of $\frac{1}{2}(J-M)$ obtained by deleting the first row and column. It is easy to see that $A$ satisfies (2), thus $A$ is the adjacency matrix of a tournament of order $n-1$.

Assuming that $M$ is a skew Hadamard matrix in the above construction, it has been shown that the existence of the following are equivalent:

1. Skew Hadamard matrices of order $n$.

2. Doubly regular tournaments of order $n-1$ [7].

3. Tournaments of order $n-1$ with eigenvalues $\frac{n-2}{2}$ (with algebraic multiplicity one) and $\frac{-1 \pm \sqrt{1-n}}{2}$ ) (each with algebraic multiplicity $\frac{n-2}{2}$ ) [2].

Recently, another characterization of a skew Hadamard matrix of order $n$ in terms of the spectrum of the Seidel matrix of a tournament of order $n-2$ was given in [5].

In this paper, a characterization of skew E-W matrices in terms of tournament is given. This characterization can be considered as the analogous one to the characterization of skew Hadamard matrices given in [7].

Notation. Throughout this paper we use $s_{x}$ for the entry of the score vector (sum row) corresponding to the row of $A$ indexed by vertex $x$ and $V^{h}=\left\{y \in V: s_{y}=h\right\}$. The notation $e \in W \times Z$ means an edge of $T=(V, E)$ defined by one vertex in $W$ and the other one in $Z$ where $W$ and $Z$ are subsets of $V$. We use $t$ for a positive integer.

\section{The Main Result}

As introduced in Sect. 1, Ehlich-Wojtas' bound provides an upper bound of the maximum determinant of all $n \times n$ matrices with elements \pm 1 when $n \equiv 2(\bmod 4)$. Moreover, equality in (1) holds if and only if there exists a $(1,-1)$-matrix $B$ of order $n$, such that

$$
B B^{T}=B^{T} B=\left(\begin{array}{ll}
L & 0 \\
0 & L
\end{array}\right)
$$


with $L=(n-2) I+2 J$ a square matrix of order $\frac{n}{2}$. In addition, any $(-1,1)$-matrix $M$ attaining Ehlich-Wojtas' bound is equivalent to a matrix $B$ satisfying (4) (see [3]).

Two matrices $M$ and $N$ are said to be Hadamard equivalent or equivalent if one can be obtained from the other by a sequence of the operations:

- interchange any pairs of rows and/or columns;

- multiply any rows and/or columns through by -1 .

In other words, we say that $M$ and $N$ are equivalent if there exist monomial matrices $P$ and $Q$ such that $P M Q=N$.

Let us point out that if $M$ is equivalent to $B$ then $B=P M Q$ where $P$ and $Q$ are monomial matrices. Thus,

$$
B B^{T}=P M Q Q^{T} M^{T} P^{T}=P M M^{T} P^{T}=\left(P M P^{T}\right)\left(P M P^{T}\right)^{T} .
$$

Hence, if $B$ satisfies (4), $P M P^{T}$ as well.

Remark 2.1. Without loss of generality, we can always assume that if $M$ reaches Ehlich-Wojtas's bound then there exists a monomial matrix $P$ such that $P M P^{T}$ satisfies (4).

Condition (4) implies some combinatorial properties, regarding the number of positive entries of the rows (resp. columns) of $B$. The rows of any $(-1,1)$-matrix of size $n$ can be classified as of even or odd type, depending on the parity of the number of +1 's that they contain. It is apparent that the inner product of two rows of the same type is congruent to 2 modulo 4, while the inner product of two rows of opposite type is congruent to 0 modulo 4 . In these circumstances, the block structure of the matrix in (4) implies that the rows from 1 to $2 t+1$ of $B$ share a common type, whereas the rows from $2 t+2$ to $4 t+2$ share the opposite type. The same argument translates to the columns of $B$. This is a main difference with usual Hadamard matrices of order a multiple of 4 , in which rows of different type cannot occur. Notice that this balanced structure of even and odd type rows does not need to be attained anymore when $2 n-2$ is not the sum of two squares.

If $B$ is skew, then $2 n-2$ is the sum of two squares where the first integer is 1 (see [1]). Moreover, assuming that $B$ is normalized, the rows of odd type can be classified into two kinds depending on their row sum and there are exactly the same number of every kind.

Lemma 2.1. Let $M$ be a skew (-1,1)-matrix of order $n=4 t+2$ and $A$ be its corresponding adjacency matrix of the tournament of order $n-1$. If $M$ is a skew $E-W$ matrix, then the entries of the score vector $A \mathbf{1}$ are $2 t, 2 t+1$ and $2 t-1$, each appearing $2 t+1$, $t$ and $t$ times, respectively.

Proof. Let $M=\left[m_{i, j}\right]$ be a skew E-W matrix of order $n=4 t+2$. We can always assume that the first row of $M$ consists entirely of +1 since multiplying row $r$ and column $r$ of $M$ by -1 the value of the determinant does not change and neither does the skew character. This implies $m_{i, 1}=-1,2 \leq i \leq n$. As $M$ is skew, $m_{i, i}=+1, \quad 1 \leq i \leq n$. Form a new matrix $A=\left[a_{i, j}\right], 1 \leq i, j \leq n-1$, by putting 


$$
a_{i, j}=\left\{\begin{array}{rr}
+1, & m_{i+1, j+1}=-1 \\
0, & m_{i+1, j+1}=+1
\end{array}\right.
$$

As it was mentioned in Sect. 1, $A$ is the adjacency matrix of a tournament of order $n-1=4 t+1$. Since $M$ is equivalent to a matrix $B$ satisfying (4), thus for $j \neq 1$,

$$
\sum_{i=1}^{n} m_{1, i} m_{j, i}=\sum_{i=1}^{n} m_{j, i}=\left\{\begin{aligned}
\pm 2, & 2 \leq j \leq 2 t+1 \\
0, & 2 t+2 \leq j \leq 4 t+2
\end{aligned}\right.
$$

so that each row of $M$ from the $2 t+2$-th until the $4 t+2$-th contains $(2 t+1)+1$ 's and $(2 t+1)-1$ 's. Thus, the last $2 t+1$ rows of $A$ contains exactly $2 t+1$ 's, so that the last $2 t+1$ entries of the score vector are equal to $2 t$ (i.e., $s_{i}=2 t, 2 t+1 \leq i \leq 4 t-1$ ). For the rows of $M$ from the 2-nd until the $2 t+1$-th, we have two cases:

1. If $\sum_{i=1}^{n} m_{j, i}=+2$, then every type of these rows of $M$ contains $(2 t+2)+1$ 's and (2t) -1 's. Thus, the corresponding rows of $A$ contain $(2 t-1)+1$ 's.

2. If $\sum_{i=1}^{n} m_{j, i}=-2$, then every type of these rows of $M$ contain $(2 t)+1$ 's and $(2 t+2)-1$ 's. Thus, the corresponding rows of $A$ contain $(2 t+1)+1$ 's.

So that the first $2 t$ entries of the score vector are equal to either $2 t-1$ or $2 t$ (i.e., $s_{i}=2 t+1$ or $\left.s_{i}=2 t-1,1 \leq i \leq 2 t\right)$.

Finally, let $\left|V^{2 t+1}\right|=\left|\left\{i: s_{i}=2 t+1\right\}\right|$ and $\left|V^{2 t-1}\right|=\left|\left\{i: s_{i}=2 t-1\right\}\right|$. We have

$$
\left\{\begin{aligned}
\left|V^{2 t+1}\right|+\left|V^{2 t-1}\right| & =2 t \\
\left|V^{2 t+1}\right|(2 t+1)+\left|V^{2 t-1}\right|(2 t-1) & =4 t^{2}
\end{aligned}\right.
$$

The second equation of the system above follows from (3). Hence, $\left|V^{2 t+1}\right|=$ $\left|V^{2 t-1}\right|=t$, and this concludes with the desired result.

Lemma 2.2. Assume the same hypothesis and notation as in Lemma 2.1. Thus, the adjacency matrix A satisfies (after permutations of row and columns)

$$
A A^{T}=\left[\begin{array}{cc}
X & Y \\
Z & W
\end{array}\right] \quad \text { and } \quad A^{T} A=\left[\begin{array}{cc}
\tilde{X} & \tilde{Y} \\
\tilde{Z} & W
\end{array}\right]
$$

where

- $X=\left[\begin{array}{ll}X_{1} & X_{2} \\ X_{3} & X_{4}\end{array}\right]$ and $\tilde{X}=\left[\begin{array}{ll}X_{4} & X_{2} \\ X_{3} & X_{1}\end{array}\right]$ with

$$
X_{1}=t I_{t}+(t-1) J_{t}, \quad X_{4}=t I_{t}+(t+1) J_{t}, \quad X_{2}=X_{3}=(t-1) J_{t} .
$$


- $Y=\left[\begin{array}{r}(t-1) J_{t, 2 t+1} \\ t J_{t, 2 t+1}\end{array}\right] \quad$ and $\quad \tilde{Y}=\left[\begin{array}{r}t J_{t, 2 t+1} \\ (t-1) J_{t, 2 t+1}\end{array}\right]$.

- $Z=Y^{T}$ and $\tilde{Z}=\tilde{Y}^{T}$.

- $W=\left[\begin{array}{ll}W_{1} & W_{2} \\ W_{3} & W_{4}\end{array}\right]$ with

$$
W_{1}=t I_{\xi}+t J_{\theta}, \quad W_{2}=W_{3}^{T}=(t-1) J_{\xi, \theta}, \quad W_{4}=t I_{\theta}+t J_{\theta},
$$

where $\xi+\theta=2 t+1$ and $\xi, \theta \geq 0$. $J_{r, s}$ denotes the all-ones matrix with r rows and $s$ columns, and $J_{r}$ denotes $J_{r, r}$. We follow the same notation for the identity matrix.

Proof. Considering the rows $(k-1)$-th and $(l-1)$-th of $A$, with $k<l$. Let

$$
\begin{aligned}
& \alpha=\left|\left\{i: m_{k, i}=m_{l, i}=+1\right\}\right|, \\
& \beta=\left|\left\{i: m_{k, i}=m_{l, i}=-1\right\}\right|, \\
& \gamma=\left|\left\{i: m_{k, i}=-m_{l, i}=+1\right\}\right|, \\
& \delta=\left|\left\{i: m_{k, i}=-m_{l, i}=-1\right\}\right| .
\end{aligned}
$$

By construction of $A, \beta-1$ (respectively, $\alpha$ ) is equal to the number of +1 's (respectively, 0 's) that rows $(k-1)$ and $(l-1)$ of $A$ contain in the same column. That is, the vertices $(k-1)$ and $(l-1)$ of $T$ jointly dominate precisely $\beta-1$ vertices and, they are jointly dominated precisely by $\alpha-1$ vertices (since either $m_{k, l}=m_{l, l}=+1$ or $\left.m_{k, k}=m_{l, k}=+1\right)$. Therefore,

$$
\left[A A^{T}\right]_{k-1, l-1}=\beta-1 \text { and }\left[A^{T} A\right]_{k-1, l-1}=\alpha-1 .
$$

Now considering the set of rows of $M$ but the first, we can classify them attending to their row sum in three types. For $2 \leq r \leq 4 t+2$

$$
\begin{aligned}
& R_{1}=\left\{r: \sum_{i=1}^{4 t+2} m_{r, i}=-2\right\}, \\
& R_{2}=\left\{r: \sum_{i=1}^{4 t+2} m_{r, i}=0\right\}, \\
& R_{3}=\left\{r: \sum_{i=1}^{4 t+2} m_{r, i}=+2\right\} .
\end{aligned}
$$

In Lemma 2.1 it was proven

1. $r \in R_{2} \Leftrightarrow 2 t+2 \leq r \leq 4 t+2$. 
2. $\left|R_{1}\right|=\left|R_{3}\right|=t$.

Attending to this classification, there are six possible cases of choosing pairs of rows.

1. Let $k \in R_{1}$ and $l \in R_{2}$. Counting the -1 and +1 in each row. We have

$$
\begin{aligned}
& \alpha+\delta=2 t+1=\beta+\gamma \\
& \beta+\delta=2 t+2 \\
& \alpha+\gamma=2 t
\end{aligned}
$$

so $\alpha+\delta=\beta+\gamma=\beta+\delta-1=\alpha+\gamma+1$, or $\delta=\gamma+1$ and $\beta=\alpha+1$.

Recall that negating certain set of rows and the same set of columns of $M$, we obtain a new matrix $B$ which satisfies (4). Hence, $\sum_{i=1}^{4 t+2} m_{k, i} m_{l, i}=0$, so $\alpha+\beta=\gamma+\delta$. Thus, $\beta=t+1$ and $\alpha=t$.

2. Let $k \in R_{3}$ and $l \in R_{2}$. By a similar argument, $\beta=t$ and $\alpha=t+1$.

3. Let $k, l \in R_{2}$. Counting the number of -1 and +1 in each row,

$$
\beta+\delta=\alpha+\gamma=2 t+1=\beta+\gamma=\alpha+\delta
$$

so $\gamma=\delta$ and $\alpha=\beta$. Also, $\sum_{i} m_{k, i} m_{l, i}= \pm 2$.

If $\sum_{i} m_{k, i} m_{l, i}=+2$, then

$$
\begin{aligned}
& \alpha+\beta=2 t+2 \\
& \gamma+\delta=2 t
\end{aligned}
$$

Thus $\beta=t+1=\alpha$.

Similarly, if $\sum_{i} m_{k, i} m_{l, i}=-2$, then $\beta=t=\alpha$.

4. Let $k \in R_{1}$ and $l \in R_{3}$. Counting the number of -1 and +1 in each row, we get $\alpha=\beta$. Since negating certain set of rows and the same set of columns of $M$, we obtain a new matrix $B$ which satisfies (4), then $\sum_{i} m_{k, i} m_{l, i}=-2$. So, $\alpha+\beta=2 t$. Hence, $\beta=t=\alpha$.

5. Let $k, l \in R_{1}$. Taking into account that $\sum_{i} m_{k, i} m_{l, i}=2$ and using an analogous argument. We get $\beta=t+2$ and $\alpha=t$.

6. Let $k, l \in R_{3}$. Taking into account that $\sum_{i} m_{k, i} m_{l, i}=2$ and using an analogous argument. We get $\beta=t$ and $\alpha=t+2$.

Consequently, taking into account the size of the sets $R_{1}, R_{2}$, and $R_{3}$, the identities (6) and the values of $\alpha$ and $\beta$, it follows the desired result.

Definition 2.1. Let $T=(V, E)$ be a tournament of order $4 t+1$, it said to be an E-W tournament if

- its adjacency matrix $A$ satisfies (after permutations of row and columns) the identities in (5).

Or equivalently, 
- The entries of its score vector $s_{i}$ are $2 t, 2 t+1$ and $2 t-1$, each appearing $2 t+1$, $t$ and $t$ times, respectively.

- Let $e \in E$.

$$
\left(d^{+}, d^{-}\right)(e)=\left\{\begin{array}{cl}
(t, t-1), & e \in V^{2 t} \times V^{2 t+1}, \\
(t-1, t), & e \in V^{2 t-1} \times V^{2 t}, \\
(t-1, t-1), & e \in V^{2 t-1} \times V^{2 t+1}, \\
(t+1, t-1), & e \in V^{2 t+1} \times V^{2 t+1}, \\
(t-1, t+1), & e \in V^{2 t-1} \times V^{2 t-1}, \\
(t, t), & e \in\{x\} \times V_{x, t}, \\
(t-1, t-1), & e \in\{x\} \times V_{x, t-1}, \\
(t, t), & e \in V_{x, t} \times V_{x, t}, \\
(t, t), & e \in V_{x, t-1} \times V_{x, t-1}, \\
(t-1, t-1), & e \in V_{x, t-1} \times V_{x, t} ;
\end{array}\right.
$$

for some $x \in V^{2 t}$, let $V_{x, d}=\left\{y \in V^{2 t}: d^{+}\left(e_{x, y}\right)=d\right\}$ such that $V_{x, t} \cup V_{x, t-1}=$ $V^{2 t} \backslash\{x\}$. Moreover, either $V_{x, t}$ or $V_{x, t-1}$ could be empty.

Remark 2.2. The equivalence above follows from the fact that the vertices $(k-1)$ and $(l-1)$ of $T$ jointly dominate precisely $\left[A A^{T}\right]_{k-1, l-1}$ vertices and, they are jointly dominated precisely by $\left[A^{T} A\right]_{k-1, l-1}$ vertices.

The following result follows immediately from Lemmas 2.1 and 2.2.

Proposition 2.1. Let $M$ be a skew $(-1,1)$-matrix of order $n=4 t+2$ and $A$ be its corresponding adjacency matrix of the tournament of order $n-1$. If $M$ is a skew E-W matrix, then the tournament with adjacency matrix $A$ is an E-W tournament.

In the next result, we will show that the converse statement holds.

Proposition 2.2. Let $T$ be a tournament of order $4 t+1$ and $A=\left[a_{i, j}\right]$ be its adjacency matrix. If $T$ is an $E-W$ tournament, then $M=\left[m_{i, j}\right]$ with

$$
m_{i, j}=\left\{\begin{array}{cl}
+1, & i=1 \\
-1, & i>1, \text { and } j=1 \\
1-2 a_{i-1, j-1}, & 2 \leq i, j \leq 4 t+2
\end{array}\right.
$$

is a skew $E-W$ matrix of order $4 t+2$.

Proof. We can always assume that the vertices of $V$ are ordered in such a way so,

$$
s_{i}=\left\{\begin{array}{cl}
2 t-1, & 1 \leq i \leq t \\
2 t+1, & t+1 \leq i \leq 2 t \\
2 t, & 2 t+1 \leq i \leq 4 t+1
\end{array}\right.
$$


We have to show that $M$ is equivalent to $B$ such that $B$ satisfies (4). To this end, consider sums of the form

$$
\sum_{i=1}^{4 t+2} m_{k, i} m_{l, i}, \quad 1 \leq k, l \leq 4 t+2 .
$$

1. $k=l$,

$$
\sum_{i=1}^{4 t+2} m_{k, i} m_{l, i}=\sum_{i=1}^{4 t+2} m_{k, i}^{2}=\sum_{i=1}^{4 t+2} 1=4 t+2
$$

2. For $k=1 \neq l$,

$$
\sum_{i=1}^{4 t+2} m_{1, i} m_{l, i}=\sum_{i=1}^{4 t+2} m_{l, i}=4 t-2 s_{l-1}=\left\{\begin{aligned}
+2, & 2 \leq l \leq t+1 \\
-2, & t+2 \leq l \leq 2 t+1 \\
0, & 2 t+2 \leq l \leq 4 t+2
\end{aligned}\right.
$$

3. $2 \leq k \leq 2 t+1$ and $2 t+2 \leq l \leq 4 t+2$.

Recall that $d^{+}\left(e_{x_{k}, x_{l}}\right)=\left|\left\{\bar{i}: a_{k, i}=a_{l, i}=+1\right\}\right|$ and $d^{-}\left(e_{x_{k}, x_{l}}\right)+1=$ $\left|\left\{i: a_{k, i}=a_{l, i}=0\right\}\right|$. Since either $\left(d^{+}, d^{-}\right)\left(e_{x_{k-1}, x_{l-1}}\right)=(t, t-1)$ or $\left(d^{+}, d^{-}\right)\left(e_{x_{k-1}, x_{l-1}}\right)=(t-1, t)$, so $\left|\left\{i: m_{k, i} m_{l, i}=1\right\}\right|=2 t+1$. Thus,

$$
\sum_{i=1}^{4 t+2} m_{k, i} m_{l, i}=(2 t+1)-(2 t+1)=0 .
$$

4. $2 \leq k \leq t+1$ and $t+2 \leq l \leq 2 t+1$. Since $\left(d^{+}, d^{-}\right)\left(e_{x_{k-1}, x_{l-1}}\right)=(t-1, t-1)$, so $\left|\left\{i: m_{k, i} m_{l, i}=1\right\}\right|=2 t$. Thus,

$$
\sum_{i=1}^{4 t+2} m_{k, i} m_{l, i}=2 t-(2 t+2)=-2 .
$$

5. $2 \leq k<l \leq t+1$ or $t+2 \leq k<l \leq 2 t+1$. In similar manner, we have

$$
\sum_{i=1}^{4 t+2} m_{k, i} m_{l, i}=(2 t+2)-2 t=2 .
$$

6. $2 t+2 \leq k<l \leq 4 t+2$. Let us take the sets $V_{x_{2 t+1}, t}$ and $V_{x_{2 t+1}, t-1}$. Consider the cases:

(a) For $k=2 t+2$.

$$
\text { If } x_{l-1} \in V_{x_{2 t+1}, t} \text {. Thus, } \sum_{i=1}^{4 t+2} m_{k, i} m_{l, i}=2 \text {. }
$$




$$
\text { If } x_{l-1} \in V_{x_{2 t+1}, t-1} \text {. Thus, } \sum_{i=1}^{4 t+2} m_{k, i} m_{l, i}=-2 \text {. }
$$

(b) For $2 t+2<k<l \leq 4 t+2$.

(i) If $e_{x_{k-1}, x_{l-1}} \in\left(V_{x_{2 t+1}, t} \times V_{x_{2 t+1}, t}\right) \bigcup\left(V_{x_{2 t+1}, t-1} \times V_{x_{2 t+1}, t-1}\right)$. Thus,

$$
\sum_{i=1}^{4 t+2} m_{k, i} m_{l, i}=2 .
$$

(ii) If $e_{x_{k-1}, x_{l-1}} \in V_{x_{2 t+1}, t-1} \times V_{x_{2 t+1}, t}$. Thus,

$$
\sum_{i=1}^{4 t+2} m_{k, i} m_{l, i}=-2 .
$$

Consequently, multiplying by -1 the rows and columns of $M$ from the $(t+2)$-th until the $(2 t+1)$-th and the corresponding rows and columns of $M$ indexed with the elements of $V_{x_{2 t+1}, t-1}$, then this new matrix denoted by $B$ satisfies (4).

By construction it is easy to see that $M+M^{T}=2 I$. This concludes the proof.

Finally, we state the main result of this paper which is an immediate consequence of Propositions 2.1 and 2.2.

Theorem 2.1. The existence of the following are equivalent:

1. Skew $E-W$ matrices of order $4 t+2$.

2. E-W tournaments of order $4 t+1$.

\section{Conclusions and Further Work}

In this paper we have proved that the existence of a skew E-W matrix of order $4 t+2$ is equivalent to the existence of an E-W tournament of order $4 t+1$. This kind of tournament has been defined attending to their vertices and edges degrees. Examples of skew E-W matrices for small orders have been provided in [1].

A characterization of $\mathrm{E}-\mathrm{W}$ matrices of order $4 t+2$ in terms of spectral data for tournaments of order $4 t+1$, as analogous to the result for skew Hadamard matrices given in [2], is a challenging problem. After looking over small orders, we conjecture that the existence of the following are equivalent:

1. Skew E-W matrices of order $n=4 t+2$.

2. Tournaments of order $n-1$ with eigenvalues:

(a) The roots of the polynomial $P(x)=-t(n-3)-\frac{n-4}{2} x^{2}+x^{3}$.

(b) $\frac{-1 \pm \sqrt{3-n}}{2}$ each with algebraic multiplicity $\frac{n-4}{2}$. 
Acknowledgements The author would like to thank the anonymous referee for his valuable comments. The author would also like to thank Kristeen Cheng for her reading of this manuscript. This work has been partially supported by the research project FQM-016 from JJAA (Spain).

\section{References}

1. Armario, J.A., Frau, M.D.: An upper bound on the maximal determinant of skew matrices of the conference type (2014, preprint)

2. de Caen, D., Gregory, D.A., Kirkland, S.J., Pullman, N.J.: Algebraic multiplicity of the eigenvalues of a tournament matrix. Linear Algebra Appl. 169, 179-193 (1992)

3. Ehlich, H.: Determiantenabschätzungen für binäre Matrizen. Math. Z. 83, 123-132 (1964)

4. Kharaghani, H., Orrick, W.: D-optimal matrices. In: Colbourn, C., Dinitz, J. (eds.) The CRC Handbook of Combinatorial Designs, pp. 296-298, 2nd edn. Taylor and Francis, Boca Raton (2006)

5. Nozaki, H., Suda, S.: A characterization of skew Hadamard matrices and doubly regular tournaments. Linear Algebra Appl. 437, 1050-1056 (2012)

6. Orrick, W., Solomon, B.: The hadamard maximal determinant problem (2005). http://www. indiana.edu/ maxdet/. Cited 3 Sept 2014

7. Reid, K.B., Brown, E.: Doubly regular tournaments are equivalent to skew Hadamard matrices. J. Comb. Theory Ser. A 12, 332-338 (1972)

8. Wojtas, W.: On Hadamard's inequallity for the determinants of order non-divisible by 4. Colloq. Math. 12, 73-83 (1964) 